\title{
Editorial
}

\section{ABT-737, proving to be a great tool even before it is proven in the clinic}

\author{
DL Vaux ${ }^{\star, 1}$ \\ Cell Death and Differentiation (2008) 15, 807-808; doi:10.1038/cdd.2008.31
}

The remarkable growth in interest in apoptosis began when inhibition of cell death was linked to human cancer, and components of the mechanism for cell death were identified, beginning with recognition that the product of the bcl-2 gene, which is activated in follicular lymphoma, is an inhibitor of cell death. ${ }^{1}$ However, the resulting avalanche of publications also brought confusion and controversy, especially when mechanisms of cell death were inferred from the morphological appearance of dying cells.

The development of the $\mathrm{Bcl}-2 / \mathrm{Bcl}-\mathrm{x}$ inhibitor ABT-737 by Abbott Inc. ${ }^{2}$ has been ground-breaking for two reasons. Firstly, if this inhibitor or related compounds are successful in human trials for the treatment of cancer, then the global effort in studying cell death will be justified as something of practical use, and not merely of academic interest. Secondly, ABT-737 provides an exquisite tool to test various models of cell death; to verify or disprove assumptions based on correlative morphological evidence.

In this issue of Cell Death and Differentiation, Vogler et al. ${ }^{3}$ provide a nice example of this second type. They used ABT737 to treat primary chronic lymphocytic leukaemia (CLL) cells, and determined the effects on Bax, Bak, and the mitochondria. Results using ABT-737 can be interpreted with much more confidence than those using other putative $\mathrm{BH} 3-$ only mimetics, because unlike most of the other compounds, Bax/Bak double knockout cells are completely unaffected by ABT-737, indicating it only induces cell death by the Bax/Bakdependent mechanism. ${ }^{4}$

Like follicular lymphoma, CLL is a malignancy of B lineage cells that is very common, not usually rapidly lethal, but rarely cured using current therapies. However, unlike follicular lymphoma, CLL cells do not bear the $t(14: 18)$ translocation that activates $\mathrm{Bcl}-2$. Nevertheless, Vogler et al. ${ }^{3}$ treated primary CLL cells with ABT-737, and saw a very rapid, and most gratifying induction of apoptosis that will be a cause for optimism among haemato-oncologists.

Vogler et al. ${ }^{3}$ then analysed the molecular and morphological effects of ABT-737 on CLL cells in detail. At low nanomolar concentrations, ABT-737 rapidly induced primary CLL cells to undergo apoptosis, accompanied by loss of cytochrome $c$ from the mitochondria, activation of caspases, condensation of the chromatin and surface exposure of phosphatidylserine (PS). While PS exposure, and presumably other caspase-dependent events, could be blocked by the caspase inhibitor zVAD-fmk, this compound would not be expected to prevent the death of the cells, as genetic experiments have shown that Bax/Bak-dependent events at the mitochondria are sufficient for cells to commit suicide without caspase activity. ${ }^{5}$ The fact that cells can kill themselves, even though apoptosis (defined morphologically and by PS exposure) is blocked, illustrates how genetic and biochemical data can provide insights beyond those of morphology.

Nevertheless, activation of Bax and Bak at the mitochondria appears to be a key event in apoptosis induced by ABT-737. Vogler et al.'s ${ }^{3}$ experiments do not address the controversy over whether $\mathrm{BH} 3$-only pro-apoptotic proteins are needed to directly bind to and activate $\mathrm{Bax}$ and $\mathrm{Bak},{ }^{6}$ or whether they simply neutralise antiapoptotic proteins $\mathrm{Bcl}-2, \mathrm{Bcl}-\mathrm{x}$ and $\mathrm{Mcl}-1 .{ }^{7}$ As a BH3-only 'mimetic', ABT-737 could be helping to inactivate the antiapoptotic proteins, or it could displace BH3-only proteins, freeing them to activate Bax and Bak.

In the ABT-737 treated cells, Bax and Bak underwent conformational changes, and Bax moved from the cytoplasm to the mitochondria. Bax and Bak then formed oligomers. It is interesting to compare appearance of these (presumably crosslinked) oligomers with those observed by other groups. Whereas Vogler et $a l^{3}$ saw Bax monomers at the expected molecular weight of $\sim 20 \mathrm{kDa}$ and Bak monomers at $\sim 30 \mathrm{kDa}$, Letai et al. $^{6}$ found that monomers of Bax and Bak both ran with apparent molecular weight of $21 \mathrm{kDa}$, and, even more curiously, Chipuk et al. ${ }^{8}$ reported that even recombinant monomeric Bax becomes dimeric when subjected to SDSPAGE. Some of these issues might be resolved if better antibodies were produced; in the paper by Letai et al. ${ }^{6}$ the antiBax and anti-Bak antibodies seemed to detect the same bands, and in Vogler et al. $^{3}$ both the anti-Bax and anti-Bak antibodies also detected prominent bands that were smaller than the monomers, and are presumably cross reacting proteins of unknown identity.

Using EM, Vogler et al. ${ }^{3}$ then determined the effects of ABT-737 (and consequent Bax/Bak activation) on the mitochondria. Here, they observed mitochondrial inner membrane permeabilisation, rapid swelling of the cristae

\footnotetext{
${ }^{1}$ Department of Biochemistry, La Trobe University, Victoria 3086, Australia

${ }^{*}$ Corresponding author: DL Vaux, Department of Biochemistry, La Trobe University, 1G Royal Parade, Bundoora Vic, Victoria, 3086, Australia.

Tel: + 613947922 11; Fax: + 613947924 67; E-mail: d.vaux@latrobe.edu.au
} 
and the mitochondria as a whole, followed by breakage of the outer mitochondrial membrane. These latter morphological changes are usually termed necrotic, rather than apoptotic. While the mechanism by which activated Bax/Bak affected the inner mitochondrial membrane was not determined, it was not caspase dependent, nor did it involve the permeability transition pore, as addition of the cyclosporin A also had no effect.

These observations using ABT-737 will be of interest to cell death researchers, who should be mindful of them before concluding from morphological appearances of the mitochondria that cell death is not occurring by the classic Bax/Bakdependent apoptotic mechanism. Haemato-oncologists will be encouraged to note that even if a cell has not translocated the bcl-2 gene, it might still be exquisitely sensitive to $\mathrm{BH} 3-$ only mimetics such as ABT-737.

1. Vaux DL, Cory S, Adams JM. Nature 1988; 335: 440-442.

2. Oltersdorf T et al. Nature 2005; 15: 677-681.

3. Vogler $\mathrm{M}$ et al. Cell Death Differ 2008; 15: 820-830.

4. van Delft MF et al. Cancer Cell 2006; 10: 389-399.

5. Ekert PG et al. J Cell Biol 2004; 165: 835-842.

6. Letai A, Bassik MC, Walensky LD, Sorcinelli MD, Weiler S, Korsmeyer SJ. Cancer Cell 2002; 2: 183-192.

7. Willis SN et al. Genes Dev 2005; 18: 1294-1305.

8. Chipuk JE et al. Science 2004; 303: 1010-1014. 